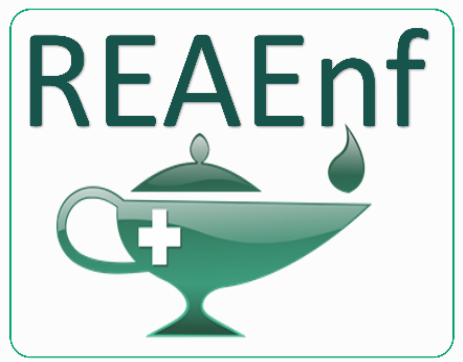

Revista Eletrônica Acervo Enfermagem
RELATO DE EXPERIÊNCIA

Recebido em: 7/2020

Aceito em: 8/2020

Publicado em: 9/2020

\title{
Ações de enfermagem na promoção da saúde e qualidade de vida de mulheres no climatério
}

Nursing actions in the promotion of health and quality of life of women in the climacteric

Acciones de enfermería en promoción de la salud y calidad de vida de las mujeres en el climaterio

Rute Silva de Oliveira Patrício ${ }^{1}$, Orácio Carvalho Ribeiro Juniorr" ${ }^{2 \star}$ Suzana Maria da Silva Ferreira ${ }^{3}$, Tatiane Silva de Araújo ${ }^{1}$, Luciane Cativo Brasil ${ }^{4}$, Jociane Martins da Silva ${ }^{4}$, Miquele Soares Barbosa $^{4}$, Antônio Victor Souza Cordeiro ${ }^{4}$, Lucas Saboia Pereira ${ }^{4}$, Murilo Henrique Nascimento Araújo ${ }^{4}$.

Resumo: Os sinais e sintomas que compõem a síndrome climatérica geram consequências que podem afetar o bem-estar da mulher, devido ao preconceito e tabus levantados pela sociedade ao longo dos anos. Objetivou-se apresentar as experiências e as atividades vivenciadas por acadêmicas de enfermagem durante a disciplina de estágio supervisionado em saúde da mulher nas ações de cuidado integral às mulheres climatéricas em um parque municipal em uma cidade do Amazonas. As ações foram realizadas mediante adesão voluntária das mulheres, que posteriormente foram acompanhadas por meio da consulta de enfermagem, guiada por instrumentos específicos, bem como, ações de educação em saúde e escuta individualizada de cada participante sobre o climatério, suas características e manejo. Considerações Finais: As atividades desenvolvidas junto a esta população mostraram-se satisfatórias para o público que acompanhou e usufruiu dos serviços disponibilizados. Para as acadêmicas, figurou a oportunidade de aplicar os conhecimentos científicos do cuidado em enfermagem numa realidade de saúde diferenciada. A assistência integral mostrou-se ponto estratégico para despertar novamente a autoestima dessas mulheres, de modo que possam se perceber como protagonistas no seu autocuidado, de modo a agir para garantir seu bem-estar e qualidade de vida.

Palavras-chave: Climatério, Enfermagem, Promoção da saúde.

Abstract: The signs and symptoms that make up the climacteric syndrome have consequences that can affect the woman's well-being due to the prejudice and taboos raised by society over the years. The objective was to present the experiences and activities experienced by nursing students during supervised training in women's health in the actions of comprehensive care of climacteric women in a municipal park in an Amazon city. The actions were carried out through voluntary adherence of the women, who were then

1 Universidade Federal do Amazonas (UFAM), Manaus - AM.

2 Universidade do Estado do Pará (UEPA), Altamira - PA. *E-mail: o_ra_cio13@hotmail.com

3 Universidade Federal de Roraima (UFRR), Boa Vista - RR.

${ }^{4}$ Centro Universitário do Norte (UNINORTE). Manaus - AM. 
followed through the nursing consultation, guided by specific instruments, as well as health education actions and individual listening of each participant on the climacteric, its characteristics and management. The activities developed with this population were satisfactory for the public who accompanied and enjoyed the services provided. For the academics, it represented the opportunity to apply the scientific knowledge of nursing care in a differentiated health reality. Comprehensive care has proved to be a strategic point to reawaken the self-esteem of these women, so that they can perceive themselves as protagonists in their self-care, in order to act to guarantee their well-being and quality of life.

Keywords: Climacteric, Nursing, Health promotion.

Resumen: Los signos y síntomas que conforman el síndrome climatérico generan consecuencias que pueden afectar el bienestar de la mujer, debido a los prejuicios y tabúes generados por la sociedad a lo largo de los años. El objetivo fue presentar las experiencias y actividades experimentadas por estudiantes de enfermería durante la disciplina supervisada de pasantías en salud de la mujer en acciones de atención integral para mujeres climatéricas en un parque municipal en una ciudad amazónica. Las acciones se llevaron a cabo mediante la adhesión voluntaria de las mujeres, que posteriormente fueron seguidas a través de la consulta de enfermería, guiadas por instrumentos específicos, así como acciones de educación en salud y escucha individualizada de cada participante sobre el climaterio, sus características y manejo. Las actividades desarrolladas con esta población resultaron ser satisfactorias para el público que siguió y disfrutó de los servicios prestados. Para los académicos, hubo una oportunidad de aplicar el conocimiento científico de la atención de enfermería en una realidad de salud diferenciada. La asistencia integral demostró ser un punto estratégico para despertar nuevamente la autoestima de estas mujeres, de modo que puedan percibirse a sí mismas como protagonistas en su autocuidado, a fin de actuar para garantizar su bienestar y calidad de vida.

Palabras clave: Climaterio, Enfermería, Calidad de vida.

\section{INTRODUÇÃO}

O climatério, evolução biológica que tem início por volta dos 45 anos, é caracterizado pela diminuição gradativa da produção hormonal, especialmente do estrogênio. Consiste numa transição gradual da fase reprodutiva para a fase não reprodutiva, que é dividida em três períodos: pré-menopausa, perimenopausa (quando há ausência da menstruação por um período de 12 meses) e pós-menopausa (definida pela parada menstrual e seguirá pelo resto da vida da mulher), sendo marcada por inúmeras mudanças de ordem biológica, psicológica e social (GARCIA NK, et al., 2013; HOFFMANN M, et al., 2015).

Os principais sintomas da síndrome climatérica incluem perda da autoestima, ondas de calor denominado fogachos, suor noturno, ressecamento vaginal, diminuição da libido, oscilações de humor, intolerância, ansiedade, depressão, hipertensão arterial e insônia. Mesmo sendo sintomas de intensidade diferentes, estes geram consequências que podem afetar o bem-estar da mulher, devido ao preconceito e tabus levantados pela sociedade ao longo dos anos. Dessa forma, as mulheres não se sentem à vontade para falar de seus incômodos e anseios, ficando vulneráveis às situações de injúria física e emocional, como a dispareunia e depressão, causadas pelo incorreto manejo dos sinais e sintomas da síndrome acima descrita (ALVES ERP, et al., 2015).

Com a elevação da expectativa de vida, torna-se evidente o iminente aumento na população brasileira formada por mulheres vivenciando as alterações desencadeadas pelo climatério, o qual acarretará em maiores demandas pelos serviços de atenção e cuidado a saúde centrados nas necessidades relacionadas ao processo em curso (VALENÇA CN e GERMANO RM, 2010). Assim, é importante salientar a necessidade de uma assistência integral a mulher no climatério que atue no âmbito da manutenção da saúde, prevenção de condições incapacitantes e promoção da qualidade de vida, com ações voltadas às necessidades desta população, estejam elas nas esferas física, social, de direito e/ou cultural (MERIGHI MAB, et al., 2013). 
Acerca de políticas públicas de saúde neste contexto, Garcia NK, et al. (2013) apontam para a inexistência de ações centradas na população feminina no climatério, pois apesar dos esforços este campo de atuação não se estabelece como prioritário, enquanto o período reprodutivo (planejamento sexual, reprodutivo e gestação) continua recebendo atenção máxima das ações governamentais. Não obstante, os profissionais de saúde comumente enfatizam os aspectos clínicos, relativos às queixas dos sintomas e acabam negligenciando as queixas subjetivas, relativas à forma como essa mulher tem enfrentado esta fase da vida, e como isso tem refletido no seu convívio com o parceiro, no meio familiar e social, bem como os impactos destas situações no seu modo de viver (ALVES ERP, et al., 2015).

Em face desta assistência fragmentada, os estudos têm mostrado que é sumário o conhecimento das mulheres sobre o climatério, restringindo-se na maioria dos casos apenas as questões referentes às alterações físicas impostas por esta fase da vida, deixando imensas lacunas de entendimento a respeito das alterações psicossociais que acontecem durante a fase climatérica (VALENÇA CN e GERMANO RM, 2010; SILVEIRA CM, et al., 2014; BISOGNIN P, et al., 2015).

Neste contexto, o enfermeiro surge como importante indutor de mudança nestas práxis de cuidado, visto que tem em seu cerne de formação a educação em saúde e o cuidado voltado às necessidades humanas básicas do ser humano, incluindo aos referentes à segurança e conforto (VALENÇA CN e GERMANO RM, 2010).

Este profissional revela-se ainda como importante colaborador no atendimento as necessidades legítimas desta população, sejam estas individuais e/ou coletivas, mediante o gerenciamento do cuidado humanizado e qualificado as mulheres em todas as fases do seu ciclo de vida; não obstante, atuando como importante agente influenciador para a adesão de hábitos e práticas que repercutem em melhorias na saúde e qualidade de vida dessas mulheres (PASQUAL KK, et al., 2015).

Assim, diante da problemática apresentada e das reflexões já iniciadas, o presente estudo teve por objetivo apresentar as experiências e as atividades vivenciadas por acadêmicas de enfermagem durante a disciplina de estágio supervisionado em saúde da mulher nas ações de cuidado integral às mulheres climatéricas em um parque municipal de uma cidade no Amazonas.

\section{RELATO DE EXPERIÊNCIA}

Trata-se de um estudo descritivo, do tipo relato de experiência, o qual aponta as ações de enfermagem no âmbito da promoção da saúde e qualidade de vida realizadas com mulheres no climatério em uma instituição voltada ao público senil de uma cidade no Amazonas. As práticas foram desenvolvidas durante a disciplina Estágio Supervisionado I, no módulo de Saúde da Mulher, do curso de Enfermagem, em julho de 2016, em um parque municipal, pertencente a uma instituição de longa permanência para idosos.

Inicialmente, foi realizada uma visita técnica para observação da realidade local, conhecimento da logística e dos serviços disponibilizados na localidade e identificação do perfil populacional dos frequentadores assíduos. Tais informações forneceram subsídios que permitiram a elaboração de um plano assistencial, no qual foram descritos os objetivos, o cronograma, a descrição dos serviços e atividades a serem oferecidos.

A população feminina foi elencada como população alvo, uma vez que as práticas foram realizadas no módulo de Saúde da Mulher, onde as mulheres em pós-menopausa demonstraram maior interesse e participação nas atividades.

Diariamente eram realizadas avaliações antropométricas, clínicas e ginecológicas. Entre os serviços disponibilizados incluíam-se aferição da pressão arterial, verificação da temperatura corporal axilar; exame de glicemia capilar de jejum (apenas para clientes com histórico familiar e situação de vulnerabilidade e/ou diagnosticadas com Diabetes); pesagem medição da altura, avaliação da circunferência da panturrilha e cálculo do índice de massa corporal (IMC). Estes serviços eram realizados, prioritariamente, como triagem para as consultas de enfermagem, a fim de identificar alterações clínicas e/ou anormalidades passíveis de investigação e intervenções de enfermagem. 
Após esta etapa, as clientes eram encaminhadas para uma sala, previamente organizada de maneira a oferecer um ambiente agradável e privativo, onde as acadêmicas, supervisionadas pelos professores, realizavam as consultas de enfermagem utilizando uma abordagem humanizada e acolhedora. No primeiro contato, por meio da escuta sensível e do diálogo aberto, as mulheres eram estimuladas a expor pensamentos, emoções, sentimentos, compartilhar histórias de vida e dificuldades enfrentadas com o climatério e a senescência (ou senilidade em alguns casos). Tal comportamento visava arrematar a empatia, fortalecer o vínculo no ambiente do consultório e permitir, as estudantes, adentrar em aspectos íntimos das mulheres sem decorrer em constrangimentos e situações embaraçosas desnecessárias.

Durante as consultas, foram aplicados roteiros individualizados para consulta de enfermagem no climatério/menopausa, a fim de nortear as questões a serem avaliadas e investigadas. Constituído por questões objetivas e subjetivas, o instrumento possibilitou a coleta de informações acerca de suas condições de saúde física, mental, de ordem ginecológica e reprodutiva; acerca do convívio social e relacionamentos interpessoais e familiares; e aspectos envolvendo a cultura e o lazer. A partir deste levantamento, foram levantados os problemas de enfermagem, selecionados os diagnósticos e propostas medidas intervencionistas a fim de promover melhorias na qualidade de vida e saúde destas mulheres.

Para encerramento das atividades semanais, foram realizados momentos de educação em saúde, ocorridos no espaço social da instituição, que contavam com a presença da população geral frequentadora das atividades matinais do parque. As temáticas foram selecionadas previamente, sendo estabelecida uma por encontro sendo priorizados os temas inseridos no cotidiano da população senil. As abordagens foram realizadas de maneira interativa e dinâmica, utilizando recursos audiovisuais, onde as acadêmicas realizavam a exposição teórica, sempre partindo de fatores disparadores elegidos pelo público, seguindo-se a realização de dinâmicas e momentos de confraternizações grupais.

\section{DISCUSSÃO}

A elaboração e aplicação de um plano assistencial foi uma estratégia que conferiu maior segurança nas atividades realizadas, com vistas ao alcance de resultados promissores, uma vez que abrangia ações passíveis de aproveitamento junto ao cenário e perfil de clientes. Diversos autores salientam que esta ferramenta cujo valor abonado é, em diversas situações, indevido, estando comumente aquém dos retornos obtidos com sua aplicação. Um planejamento adequado permite o estabelecimento de parâmetros a serem alcançados baseados nas necessidades da clientela atendida, os quais asseguram adaptação das redes de atenção e serviços a realidade de saúde vivenciada e qualidade na assistência oferecida (HAUSMANN M e PEDUZZI M, 2009).

A oportunidade para aplicação do conhecimento científico do cuidado numa realidade de saúde diferenciada configurou-se numa experiência singular para as acadêmicas. A escolha desta instituição como palco das práticas desmistificou a perspectiva equivocada acerca da atenção em saúde à população feminina, cuja visão biomédica de assistência ainda perdura como principal medida resolutiva aos problemas de saúde enfrentados. A realidade das mulheres assistidas demonstrou que as condições para a prestação do cuidado são passíveis de adaptação e readaptação mediante as inclinações, apontamentos, valores e identidade sociocultural da mulher a ser assistida (CARNEIRO ACLL, et al., 2012).

O cenário utilizado permitiu a vivência de situações diferenciadas no que compete ao cuidado de mulheres no climatério. Estas experiências conduziram a uma desconstrução do raciocínio exclusivamente tecnicista, o qual está centrado na sintomatologia e que exclui outros aspectos da vida da mulher, despertando as estudantes para o aspecto humanizado da assistência, onde a empatia, o respeito e a sensibilidade empregados durante $o$ atendimento das clientes, podem auxiliar no alcance de resultados superiores aqueles que outrora eram encarados como satisfatórios. O desenvolvimento das práticas mediante essa visão humanista resulta em mudanças positivas de vínculo, interação e acolhimento, e contribui para diminuição das chances de não adesão ou abandono da terapêutica de saúde proposta (CAMILLO SO e MAIORINO FT, 2012). 
A síndrome climatérica é caracterizada por manifestações sintomáticas, cuja intensidade e manifestação diferem de uma mulher para outra, as quais exercem influência significativa sobre a capacidade de enfrentamento pessoal, pois o preconceito e os tabus disseminados na sociedade, continuam a moldar as percepções individuais e coletivas acerca desta etapa da vida feminina, levando as mulheres que estão vivenciando o climatério a sentirem-se constrangidas e desencorajadas a externar os incômodos e anseios experimentados, deixando-as vulneráveis a situações de injúria física e emocional, como a dispareunia e depressão, causadas pelo incorreto manejo dos sinais e sintomas da síndrome acima descrita (ALVES ERP, et al., 2015).

Os achados acerca desta transição, comumente, enfatizam a influência negativa que o climatério tem sobre a qualidade de vida física e emocional das mulheres. Muitos relatos apontam para a existência de individuas que vivenciam esta transição em silêncio, ou pela falta de informação e conhecimento (acham que suas queixas são incômodas) ou por acreditarem que os desconfortos e angústias surgidos durante este período não podem ser amenizados. Nesse sentido é muito importante a orientação de um profissional que tenha plena capacidade de trabalhar a educação em saúde e de acolher a mulher nos diversos estágios que o caracterizam (BISOGNIN P, et al., 2015).

$\mathrm{Na}$ rotina de atendimentos, é comum os profissionais de saúde priorizarem os aspectos clínicos, relativos às queixas sintomáticas, e negligenciarem as queixas subjetivas, relativas à forma como a mulher enfrenta esta fase da vida e como isso reflete no seu convívio com o parceiro, no meio familiar e social, bem como os impactos destas situações no seu modo de viver (ALVES ERP, et al., 2015). Em face desta assistência fragmentada, os estudos têm mostrado que é sumário o conhecimento das mulheres sobre o climatério, o qual se restringe, na maioria dos casos, as alterações físicas impostas por esta fase da vida, deixando imensas lacunas de entendimento a respeito das alterações psicossociais que acontecem durante a fase climatérica (VALENÇA CN e GERMANO RM, 2010; SILVEIRA CM, et al., 2014; BISOGNIN P, et al., 2015).

Ante isso, surge a necessidade do trabalho multidisciplinar, o qual permite o levantamento de informações detalhadas acerca do enfrentamento, estado de saúde e transformações biopsicossocioculturais enfrentadas pelas mulheres nesta fase de vida, e permite transformá-las em agentes ativos capazes de refletir e falar sobre suas percepções acerca do momento vivenciado (CAMILLO SO e MAIORINO FT, 2012). A atenção holística e humanizada, as mulheres senis, mostra-se como uma importante ferramenta de reflexão social, a qual pode vir contribuir para redução dos impactos gerados pelo processo de senescência, que resulta em inúmeras alterações físicas e psíquicas, tanto no contexto pessoal, quanto no familiar e social (SANTOS PB e SAUTHIER M, 2012). Por meio da abordagem interdisciplinar é possível contribuir para mudanças de crenças negativas a respeito do envelhecimento e fornecer esclarecimentos para dúvidas existentes quanto ao processo de senescência e senilidade feminina, incluindo as questões envolvendo o climatério e seus desdobramentos (FREITAS ER, et al., 2016).

O diálogo franco acompanhado da escuta sensível durante a consulta de enfermagem demonstra ser uma abordagem de acolhimento eficaz. A postura adotada desperta empatia nas clientes, facilitando o processo de verbalização das situações vivenciadas no contexto atual de vida, as quais, outrora, não são compartilhadas a familiares e amigos, bem como a expressão de emoções e pensamentos, comumente reprimidos, e o intercâmbio de conhecimentos e experiências (CAMILLO SO e MAIORINO FT, 2012).

A educação em saúde individual constitui uma ferramenta primordial neste contexto, a qual pode ser aplicada para transmissão de informações e compartilhamento de experiências, pois sua essência objetiva estabelecer o indivíduo como agente promotor de sua saúde através de um referencial coletivo de conhecimentos, os quais atuam em conjunto para estimular a autonomia do indivíduo na promoção de sua saúde (FIGUEIRA AB, et al., 2013).

O enfermeiro possui competência para utilizar estratégias de educação em saúde a fim de direcionar o autocuidado para as necessidades enfrentadas durante o climatério, como uma proposta de participação ativa capaz de gerar impactos positivos sobre a saúde feminina (VIDAL CRPM, et al., 2012). Faz-se necessário, portanto, que este profissional no âmbito de suas atribuições, aproprie-se de estratégias que o 
auxiliem no desenvolvimento de ações que estimulem as mulheres a atuarem como protagonistas de sua saúde, mediante a valorização do autocuidado e adesão a hábitos e práticas cotidianas que resultarão em melhorias na saúde e qualidade de vida dessas clientes (PASQUAL KK, et al., 2015).

Mediante esta concepção, pode-se inferir que as atividades desenvolvidas junto a esta população se mostraram satisfatórias para o público que acompanhou e usufruiu dos serviços disponibilizados, bem como para as acadêmicas que puderam aplicar o conhecimento científico do cuidado em enfermagem por meio de abordagens outrora não valorizadas.

As atividades desenvolvidas junto a às mulheres climatéricas mostraram-se satisfatórias para o público que acompanhou e usufruiu dos serviços disponibilizados. Para as acadêmicas, figurou a oportunidade de aplicar os conhecimentos científicos do cuidado em enfermagem numa realidade de saúde diferenciada. É notável a necessidade que as mulheres têm de falar sobre as transformações pelas quais estão passando, elas precisam ser ouvidas e direcionadas para alcançarem uma melhor qualidade de vida. Durante 0 atendimento, identificamos que é fundamental desenvolver um trabalho interdisciplinar habilitado ao fornecimento de informações detalhadas acerca do estado de saúde e das transformações na vida das mulheres nessa etapa, colocando-as numa posição ativa, capacitando-as a refletir e falar sobre suas percepções quanto ao momento vivenciado.

\section{REFERÊNCIAS}

1. ALVES ERP, et al. Climatério: a intensidade dos sintomas e o desempenho sexual. Texto Contexto Enfermagem, 2015; 24 (1): 64-71.

2. BISOGNIN P, et al. O climatério na perspectiva de mulheres. Enfermería Global, 2015; 14 (3): 168-180.

3. CAMILLO SO, MAIORINO FT. A importância da escuta no cuidado de enfermagem. Cogitare Enfermagem, 2012; 17 (3): 549-555.

4. CARNEIRO ACLL, et al. Educação para a promoção da saúde no contexto da atenção primária. Revista Panamericana Saúde Pública, 2012; 31 (2): 115-120.

5. FIGUEIRA AB, et al. Visão do enfermeiro frente à prática da educação em saúde no ambiente hospitalar. Cogitare Enfermagem, 2013; 18 (2): 310-316.

6. FREITAS ER, et al. Educação em saúde para mulheres no climatério: impactos na qualidade de vida. Reprodução e Climatério, 2016; 31 (1): 37-43.

7. GARCIA NK, et al. Ações de atenção primária dirigidas às mulheres de 45 a 60 anos de idade. Revista Eletrônica de Enfermagem, 2013; 15 (3): 713-721.

8. HAUSMANN M, PEDUZZI M. Articulação entre as dimensões gerencial e assistencial do processo de trabalho do enfermeiro. Texto Contexto Enfermagem, 2009; 18 (2): 258-265.

9. HOFFMANN M, et al. Padrões alimentares de mulheres no climatério em atendimento ambulatorial no Sul do Brasil. Ciência Saúde Coletiva, 2015; 20 (5): 1565-1574.

10. MERIGHI MAB, et al. Mulheres idosas: desvelando suas vivências e necessidades de cuidado. Revista da Escola de Enfermagem da USP, 2013; 47 (2): 408-414.

11. PASQUAL KK, et al. Atenção à saúde da mulher após os 50 anos: vulnerabilidade programática na Estratégia Saúde da Família. Revista Gaúcha de Enfermagem, 2015; 36 (2): 21-27.

12. SANTOS PB, SAUTHIER M. As evidências sobre o cuidado ético de enfermagem à mulher idosa. Revista Enfermagem UERJ, 2012; 20 (esp.2): 825-830.

13. SILVEIRA CM, et al. A mulher e o climatério: o conhecimento em questão. Revista Ricien, 2014; 4 (10): $12-17$.

14. VALENÇA CN, GERMANO RM. Concepções de mulheres sobre menopausa e climatério. Revista Rene, $2010 ; 11$ (1): 161-171.

15. VIDAL CRPM, et al. Mulher climatérica: uma proposta de cuidado clínico de enfermagem baseada em ideias freireanas. Revista Brasileira de Enfermagem, 2012; 65 (4): 680-684. 